\title{
$\angle$ Contemporary treatment of ruptured intracranial aneurysms: perspectives from the Barrow Ruptured Aneurysm Trial
}

\author{
Peter S. Amenta, MD, John D. Nerva, MD, and Aaron S. Dumont, MD \\ Department of Neurosurgery, Tulane Center for Clinical Neurosciences, Tulane University School of Medicine, New Orleans, \\ Louisiana
}

A LTHOUGH many studies have helped to shape the treatment of ruptured intracranial aneurysms (IAs), 2 specific studies have had an enormous impact on the field. The original publications of the International Subarachnoid Aneurysm Trial ${ }^{9}$ (ISAT) and the Barrow Ruptured Aneurysm Trial ${ }^{7}$ (BRAT) have unequivocally changed the treatment of ruptured IAs. Like many pivotal studies, they have garnered substantial attention and have been the subject of intense scrutiny. In the present article, Spetzler et al. ${ }^{13}$ present the 10 -year analysis of patients harboring ruptured saccular aneurysms enrolled in BRAT.

BRAT focused on 3 key outcome measures: clinical outcome, rate of retreatment, and rate of rebleeeding. Spetzler et al..$^{13}$ demonstrated that there was no stastically significant difference in clinical outcome (defined by death or modified Rankin Scale [mRS] score $>2$ ) between the 2 treatment arms through 10 years of follow-up. After the initial hospitalization, retreatment was required in 2 of 241 $(0.8 \%)$ clipped saccular aneurysms compared to 23 of 115 (20\%) coiled saccular aneurysms. Furthermore, at the 10year follow-up, complete obliteration rates were higher in the clipped compared to coiled group (50/54 [93\%] vs 5/23 [22\%]). Two patients experienced documented rebleeding and both were assigned to and treated in the coiled group (one was from the target aneurysm and another from an incidental, coiled basilar artery aneurysm).

Differences between the design of ISAT and BRAT have previously been discussed in detail ${ }^{3-6,8}$ and the respective study designs have strengths and limitations. Briefly, ISAT was a randomized trial comparing patients with ruptured IAs deemed suitable for treatment with either microsurgical clipping or endovascular coiling. As a result, the population of patients enrolled was highly se- lected, with $78 \%$ of screened patients being excluded. ${ }^{9}$ On the other hand, BRAT was designed to be an inclusive trial randomizing eligible patients to microsurgery or endovascular therapy, but allowing cross-over if the particular aneurysm was not deemed amenable for treatment with the assigned method. From the present BRAT report, ${ }^{13} 1$ of $178(<1 \%)$ clip-assigned patients crossed over to coiling, whereas 64 of $178(36 \%)$ coil-assigned patients crossed over to clipping. The data were analyzed in an intent-totreat fashion such that the coil-assigned patients crossing over to clipping were included in the coiled group for purposes of outcome. The high rate of cross-over in BRAT is an area of significant concern and is a limitation inherent in the study design.

The readership is aware that patients in BRAT were enrolled between 2003 and 2007. Certainly, much has changed in endovascular technology and innovation since the period of enrollment (over 1 decade), yet microsurgical clipping has remained relatively stable with few major incremental improvements. The proportion of patients with ruptured IAs undergoing endovascular treatment has increased sharply. ${ }^{12}$ At many centers throughout the world, $70 \%-90 \%$ (or higher) of patients harboring ruptured aneurysms are treated with endovascular methods. ${ }^{1}$ As a result, evaluation of the BRAT data in the setting of the present environment can be difficult, particularly in regard to the disparity in cross-overs between the groups.

Cross-overs from coil-assigned patients to clipping occurred with high frequency even in the very skilled hands of the Barrow Neurological Institute team of endovascular surgeons. Similarly, it should be taken into consideration that only a single patient crossed over from the clip-assigned group to the coiling group. Not only is this disparity striking from a statistical perspective, it also probably 
does not accurately reflect current practice or the way in which IA management is now evaluated. Is a ruptured wide-necked ophthalmic aneurysm in a patient with normal vision really better managed with clipping and the surgical adjuncts of clinoid removal and cervical carotid exposure? Many would probably argue that these lesions are more suitable for balloon-assisted coiling. A contemporary study may have considerably less cross-over from the coil-to-clip group and significantly greater cross-over from the clip-to-coil group.

In this report of the 10-year follow-up of BRAT, the authors have selected to examine patients with ruptured saccular IAs. The authors state that they did so because every other randomized trial comparing clipping and coiling was limited to only saccular aneurysms. At first glance, this appears to be very reasonable. However, this introduces potential problems to the analysis. First, BRAT was designed to be a study of a cohort of nonselected patients with subarachnoid hemorrhage (SAH). The present analysis is hence an analysis of a subgroup that was not predefined in the trial design (redefines a population for outcome analysis). Second, the definition of saccular versus nonsaccular aneurysm can be subjective and introduces potential bias. When the authors compared analysis of patients harboring saccular aneurysms versus all types of aneurysms with 6 years of follow-up ${ }^{14,15}$ for posterior circulation lesions coiling was associated with better outcomes if all aneurysms were included, whereas no significant differences in outcomes were observed when analyzing only saccular aneurysms. It is not clear if such differences in outcome would be seen at 10 years if all patients with aneurysms were included in this analysis.

In terms of obliteration during the follow-up period, it is not unexpected that more clip-treated patients had complete obliteration compared to coil-treated patients (50/54 [93\%] vs 5/23 [22\%], respectively). However, it should be noted that the 10-year angiographic follow-up is extremely limited. Similarly, the rate of retreatment after discharge from the hospital was higher in the coiled compared to the clipped group (23/115 [20\%] vs $2 / 241[<1 \%]$, respectively) although the indications for retreatment were not clearly delineated.

The rebleeding rates observed were low overall. Although no rebleeding was observed at 6 years of followup, ${ }^{14} 2$ SAHs were seen during 10 years of follow-up (1 from a previously ruptured, previously coiled lesion and 1 from a previously treated but unruptured lesion).

A general caveat to the obliteration, retreatment, and rebleeding data presented is that these data apply to follow-up after the initial discharge. It should be remembered that 7 patients assigned to the coil group and 5 patients assigned to the clip group ( 9 patients who actually underwent clipping and 3 patients who actually underwent coiling) were retreated by the time of discharge from the hospital after treatment of the initial hemorrhage. Additionally, rebleeding occurred after treatment during the initial hospitalization in 2 patients (both of whom underwent surgical clipping-1 assigned to the clipping group and 1 assigned to the coil group). ${ }^{7}$

There are several possibilities for the apparent lack of difference in outcomes between the coil-treated versus clip-treated patients at 10 years. First, there may truly be no difference in long-term outcome between the groups, and it may be true that the treatments are equivalent with respect to clinical outcome (although there are noted differences in complete obliteration, retreatment, and rebleeding in this report). Second, the study is underpowered to detect such differences, especially as the number of patients available for follow-up decreases and the outcome confidence intervals widen. Third, the outcome measures used are potentially not sensitive enough to detect true differences in outcome that affect a given patient's functional capacity. Although the mRS is a commonly used clinical outcome measure, it is not without its limitations. The BRAT did not include cognitive outcomes measures. Cognitive outcomes may explain differences between those patients who can return to their previous level of function and those who cannot. Data from ISAT demonstrates that cognitive impairment occurred in one-third of patients who were not otherwise disabled according to the mRS. ${ }^{11}$ Moreover, in the neuropsychological substudy from ISAT, cognitive outcomes were better in patients undergoing coiling compared to clipping. ${ }^{11}$ Of note, ISAT is the only study to compare cognitive outcomes of patients undergoing clipping versus coiling in a randomized population.

The long-term follow-up from ISAT demonstrates that the probability of death or dependency was significantly greater in the neurosurgical group compared to the endovascular group. The rate of rebleeding was higher in the endovascular group, although the risk was still low, and the probability of disability-free survival was significantly higher in the endovascular compared to the neurosurgical group at 10 years. ${ }^{10}$

The authors do not include a table examining outcomes in patients with saccular aneurysms by actual treatment, which they did include in the 6-year follow-up data published last year. ${ }^{15}$ In the prior report, there were trends from outcome at discharge through 6 years that patients who were assigned to coiling and underwent coiling had a lower incidence of poor outcome (mRS score $>2$ ) compared to patients assigned to clipping and who underwent clipping. Patients who were assigned to coiling and underwent clipping had a trend toward worse outcomes at all time points. It should be considered that these IAs not deemed suitable for endovascular therapy may represent a more complex subset of lesions (i.e., wide neck, incorporation of parent vessel, large size) or be associated with hemorrhage requiring evacuation, thus making treatment by any modality inherently more difficult and prone to complication or inherently associated with an increased probability of poor outcome. This, of course, would negatively impact the outcome in those crossing over from coiling to clipping.

The BRAT has significant strengths, including but not limited to its inclusive design, attempt to reflect everyday clinical practice, predefined outcome measures, and its thoughtful design and data reporting. It presents a single center's outcomes in managing patients with ruptured IAs in a randomized design that allowed cross-overs. It should also be noted that the study applies to a specific population of patients treated at a world-class center with the staffing, ancillary services, and resources required to treat this dis- 
ease. The surgeons are also composed of an expert team of microsurgeons and endovascular surgeons that may not be representative of treating teams elsewhere.

There are lingering questions regarding the treatment of ruptured IAs that may be addressed in future studies. ISAT-II has been proposed to address some of the remaining limitations and gaps in knowledge. ISAT-II is designed as a pragmatic, multicenter, randomized trial comparing clinical outcomes for patients not eligible for the prior ISAT. ${ }^{2}$ It will remain to be seen whether ISAT-II can be successfully completed given the perceived superiority of coiling in the minds of many clinicians who treat patients with ruptured IAs.

In summary, the present study presenting the 10-year follow-up of patients enrolled in BRAT is an important contribution to the literature. BRAT, with its inherent strengths and limitations, has helped to shape contemporary treatment of ruptured IAs. Furthermore, BRAT again demonstrates the apparent continued superiority of clipping in terms of achieving complete IA obliteration, even though most aneurysm residuals did not appear to be clinically significant. This discrepancy is the foundation of the fundamental question, "What constitutes adequate treatment of a ruptured IA?" This important question and others remain and will hopefully be addressed by future studies. In the meantime, clinicians treating patients harboring ruptured IAs will use the available data and their own judgment to provide the best possible treatment for each patient with aneurysmal SAH.

https://thejns.org/doi/abs/10.3171/2018.10.JNS182445

\section{References}

1. AlMatter M, Bhogal P, Aguilar Pérez M, Hellstern V, Bazner $\mathrm{H}$, Ganslandt $\mathrm{O}$, et al: Evaluation of safety, efficacy and clinical outcome after endovascular treatment of aneurysmal subarachnoid hemorrhage in coil-first setting. A 10-year series from a single center. J Neuroradiol 45:349-356, 2018

2. Darsaut TE, Jack AS, Kerr RS, Raymond J: International Subarachnoid Aneurysm Trial - ISAT part II: study protocol for a randomized controlled trial. Trials 14:156, 2013

3. Darsaut TE, Raymond J: Barrow Ruptured Aneurysm Trial. J Neurosurg 117:378-380, 2012 (Letter)

4. Darsaut TE, Raymond J: Barrow Ruptured Aneurysm Trial: 3-year results. J Neurosurg 119:1642-1644, 2013

5. Lanzino G: Editorial. The Barrow Ruptured Aneurysm Trial. J Neurosurg 116:133-134, 2012
6. Macdonald RL: Editorial. Clip or coil? Six years of followup in BRAT. J Neurosurg 123:605-607, 2015

7. McDougall CG, Spetzler RF, Zabramski JM, Partovi S, Hills NK, Nakaji P, et al: The Barrow Ruptured Aneurysm Trial. J Neurosurg 116:135-144, 2012

8. Molyneux A, Kerr R, Birks J: Editorial. Barrow Ruptured Aneurysm Trial. J Neurosurg 119:139-141, 2013

9. Molyneux A, Kerr R, Stratton I, Sandercock P, Clarke M, Shrimpton J, et al: International Subarachnoid Aneurysm Trial (ISAT) of neurosurgical clipping versus endovascular coiling in 2143 patients with ruptured intracranial aneurysms: a randomised trial. Lancet 360:1267-1274, 2002

10. Molyneux AJ, Birks J, Clarke A, Sneade M, Kerr RS: The durability of endovascular coiling versus neurosurgical clipping of ruptured cerebral aneurysms: 18 year follow-up of the UK cohort of the International Subarachnoid Aneurysm Trial (ISAT). Lancet 385:691-697, 2015

11. Scott RB, Eccles F, Molyneux AJ, Kerr RS, Rothwell PM, Carpenter K: Improved cognitive outcomes with endovascular coiling of ruptured intracranial aneurysms: neuropsychological outcomes from the International Subarachnoid Aneurysm Trial (ISAT). Stroke 41:1743-1747, 2010

12. Smith GA, Dagostino P, Maltenfort MG, Dumont AS, Ratliff $\mathrm{JK}:$ Geographic variation and regional trends in adoption of endovascular techniques for cerebral aneurysms. J Neurosurg 114:1768-1777, 2011

13. Spetzler RF, McDougall CG, Zabramski JM, Albuquerque FC, Hills NK, Nakaji P, et al: Ten-year analysis of saccular aneurysms in the Barrow Ruptured Aneurysm Trial. J Neurosurg [epub ahead of print March 8, 2019. DOI: 10.3171/2018.8.JNS181846]

14. Spetzler RF, McDougall CG, Zabramski JM, Albuquerque FC, Hills NK, Russin JJ, et al: The Barrow Ruptured Aneurysm Trial: 6-year results. J Neurosurg 123:609-617, 2015

15. Spetzler RF, Zabramski JM, McDougall CG, Albuquerque FC, Hills NK, Wallace RC, et al: Analysis of saccular aneurysms in the Barrow Ruptured Aneurysm Trial. J Neurosurg 128:120-125, 2018

\section{Disclosures}

The authors report no conflict of interest.

\section{Correspondence}

Aaron S. Dumont: adumont2@tulane.edu.

INCLUDE WHEN CITING

Published online March 8, 2019; DOI: 10.3171/2018.10.JNS182445. 